\title{
On the design of enhancement cavities for second harmonic generation
}

\author{
Tim Freegarde ${ }^{\mathrm{a}, *}$, Claus Zimmermann ${ }^{\mathrm{b}}$ \\ a Dipartimento di Fisica, Università di Trento, Via Sommarive 14, 38050 Povo (TN), Italy \\ ${ }^{\mathrm{b}}$ Physikalisches Institut, Eberhard Karls Universität, D-72076 Tübingen, Germany
}

Received 24 July 2001; accepted 25 September 2001

\begin{abstract}
Enhancement cavities using off-axis spherical mirrors allow the elliptical Gaussian beams from semiconductor diode lasers to be matched directly to the optimum beam profile for frequency conversion in a nonlinear crystal. We give a general recipe for the design of such cavities. (c) 2001 Elsevier Science B.V. All rights reserved.
\end{abstract}

\section{Introduction}

The use of resonant cavities to enhance the intensity of fundamental radiation for second harmonic generation has allowed the technique of frequency doubling, originally confined to pulsed and large-frame lasers, to be applied efficiently to low power continuous-wave laser diodes. Indeed, by using the harmonic itself as the source for a second doubling stage, frequency quadrupling of these tiny devices has become a reality. Whilst the design of appropriate enhancement cavities in principle requires only textbook physics, however, it is in practice complicated by off-axis astigmatism and Brewster-angle refraction. Many researchers have therefore simplified the exercise by seeking solutions with a certain symmetry, such as a cir-

\footnotetext{
${ }^{*}$ Corresponding author. Fax: +39-0461-881-696.

E-mail address: tim.freegarde@physics.org (T. Freegarde).
}

cular beam waist at the primary or secondary focus, and either accept imperfect mode matching or introduce external beam-shaping components.

A travelling-wave ring resonator is generally preferred to a simple linear cavity for several reasons. First, no light is directly reflected back to the laser to disturb its stability. Second, only a single pass through the nonlinear crystal contributes to the round-trip loss, and the harmonic is generated unidirectionally. Third, the fundamental and harmonic beams may be coupled to the cavity through plane optical components.

The use of off-axis spherical mirrors in optical resonators containing other astigmatism-inducing components - specifically, dye cells at Brewster's angle - was considered in the early days of the laser by Kogelnik et al. [1], whose description of the physics of the situation would be hard to surpass. Dunn and Dunn [2] subsequently extended the treatment to the case of a ring laser with intracavity frequency doubling crystal at Brewster's 
angle, and others have since considered related arrangements in some detail $[3,4]$. Sun et al. have recently addressed the calculation of beam parameters for a given cavity specifically for the generation of diode laser harmonics [5]. However, to our knowledge, a usable and general recipe for the design of such resonators has yet to be published.

In this paper, we describe the design of enhancement cavities that match an arbitrarily elliptical beam such as that emitted by a laser diode, via an off-axis spherical mirror, to the optimal beam for second harmonic generation, which in general is also elliptical. We consider the case of a four-mirror optical cavity composed of two plane mirrors and two concave mirrors of equal curvature, although our results contain those for a linear resonator as a special case. We allow for inclination of the frequency-doubling crystal either within or perpendicular to the resonator plane, and consider explicitly the specific cases of normal and Brewster's angle incidence. Starting from the ellipticity of the fundamental laser beam, an arbitrary choice of mirror curvature and the desired beam profile in the crystal, whose determination we have addressed in a previous paper [6], we provide a recipe by which dimensions of a suitable resonator may be calculated.

\section{Resonator geometry}

A typical four-mirror, "bow-tie" enhancement cavity is shown in Fig. 1. The nonlinear crystal is enclosed at the 'primary' beam waist mid-way between a pair of off-axis spherical concave mirrors, whose inclination defines the tangential (azimuthal) coordinates $(x, z)$ that are locally aligned with $z$ parallel to the ray axis. Two plane mirrors, of which one is usually the input coupler, then complete the ring cavity. In Fig. 1, the crystal is inclined in the tangential plane.

We begin by defining the physical dimensions and characteristics of the cavity and its components. The crystal, of thickness $t$ measured perpendicular to its faces, is presumed to have a refractive index $\eta$, and to be inclined with respect to the ray axis at external and internal angles $\theta$ and

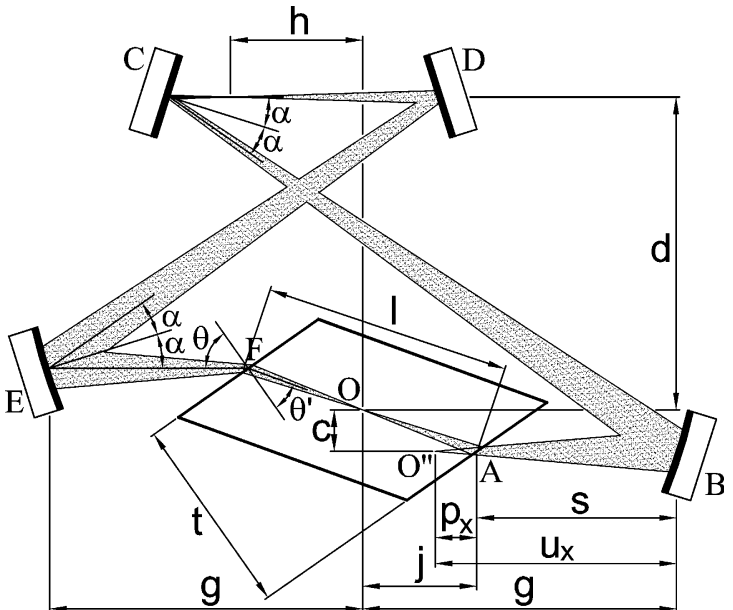

Fig. 1. Schematic layout of the enhancement cavity shown in plan view. The crystal is here shown inclined at Brewster's angle in the tangential orientation. $\mathrm{O}^{\prime \prime}$ is the apparent position of the focus when viewed from outside the crystal.

$\theta^{\prime}$. The concave mirrors, with focal length $f$ at normal incidence, make an angle $\alpha$ with the ray axis. The positions of the two plane mirrors are unimportant so long as they complete the cavity and provide the appropriate round-trip distance, but in the geometry shown they are also inclined at an angle $\alpha$ in the tangential plane.

The crystal may alternatively be inclined in the sagittal $(y, z)$ direction. Strictly, this renders the resonator nonplanar and thus requires a rather more complex analysis, not least because the modes are elliptically polarized [7]. In many practical cases, however, the distortions introduced will be small. The dimensions appropriate to the sagittally inclined geometry are shown in Fig. 2.

Although the bow-tie resonator of Fig. 1 has been by far the most popular, our analysis is also valid for the alternative cavity forms shown in Fig. 3 , with the corresponding dimensions as indicated.

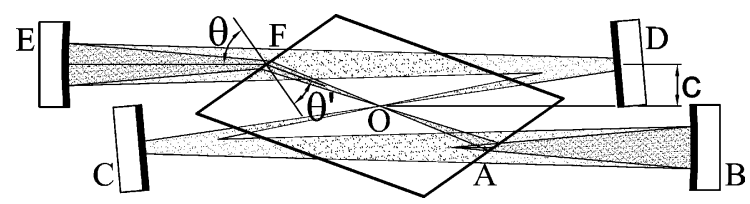

Fig. 2. Side view of the enhancement cavity showing dimensions appropriate when the crystal is inclined sagittally. 

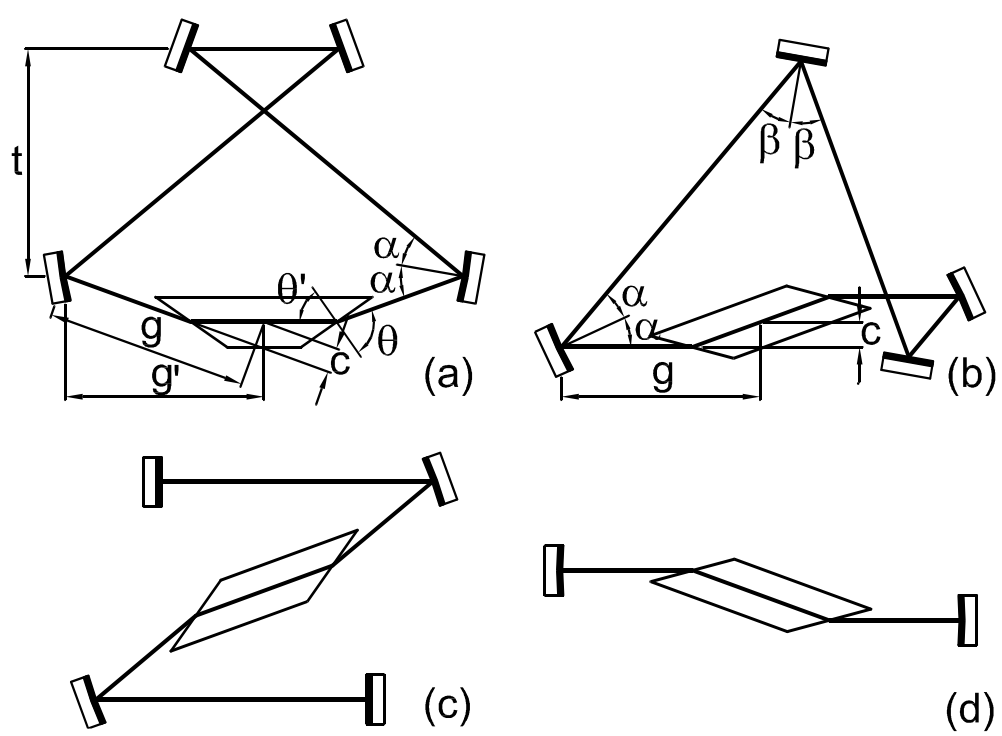

(d)

Fig. 3. Alternative cavities which permit compensation of coma: (a) ring resonator with Brewster's angle crystal faces oppositely inclined; (b) ring resonator with concave mirrors oppositely inclined; (c) symmetrical Z-shaped standing-wave cavity; (d) simple linear cavity, which corresponds to (c) when $\alpha=v=0$. The concave mirrors, which in each case are the nearest to the crystal, have focal length $f$ except in case (d), when the focal length is $f^{\prime}=2 f$.

Cavities (a) and (b) are further implementations of the ring cavity; (c) is a symmetrical four-mirror standing-wave resonator; and (d) is a simple symmetrical two-mirror linear resonator that corresponds to a special case of cavity (c).

In Appendix A, we have derived ray-transfer matrices describing various parts of the cavity round trip. These are summarized in Table 1.

\section{Design procedure}

Design of the enhancement cavity starts with the choice of crystal material, dimensions and cut, which in turn define the best Gaussian beam for harmonic generation. Knowing this beam within the crystal, we calculate the corresponding external form and the apparent optical thickness of the crystal. These parameters, together with the ellipticity of the incoming laser beam, then become the target solutions for a pair of equivalent free-space cavities corresponding to the tangential $(x, z)$ and sagittal $(y, z)$ directions and, with the addition of an assumed mirror curvature, are sufficient to establish the resonator dimensions by numerical solution. Finally, we convert the optical dimensions to the physical positions and orientations of the mirrors.

\subsection{Selecting the nonlinear crystal}

Crystals are generally chosen on the basis of their transparency and damage thresholds at the fundamental and harmonic wavelengths, the requirement of phase matching, the likely conversion efficiency and, at high powers, the susceptibility to damage and thermally induced dephasing. The efficiency depends upon the nonlinearity and Poynting vector walk-off together with the crystal dimensions, which are usually constrained by availability and cost. Calculation of the theoretical conversion efficiency has been thoroughly described elsewhere $[6,8]$ and will not be repeated here; for its absolute calculation, it will be necessary to assume the intensity enhancement due to the cavity, which depends upon the mirror reflectivity and cavity losses and will typically be a factor of between 20 and 100. Whether or not a 
Table 1

Transfer matrices describing beam propagation for the tangential and sagittal directions of the cavity ${ }^{\mathrm{a}}$

\begin{tabular}{|c|c|c|}
\hline & Tangential & Sagittal \\
\hline $\mathbf{M}_{\mathrm{OA}}^{\mathrm{ni}}$ & $\left(\begin{array}{cc}1 & \frac{t}{2 \eta} \\
0 & 1\end{array}\right)$ & $\left(\begin{array}{cc}1 & \frac{t}{2 \eta} \\
0 & 1\end{array}\right)$ \\
\hline $\mathbf{M}_{\mathrm{OA}}^{\mathrm{Bt}}$ & $\left(\begin{array}{cc}\frac{1}{\eta} & 0 \\
0 & \eta\end{array}\right)\left(\begin{array}{cc}1 & \frac{t \sqrt{1+\eta^{2}}}{2 \eta^{2}} \\
0 & 1\end{array}\right)$ & $\left(\begin{array}{cc}1 & \frac{t \sqrt{1+\eta^{2}}}{2 \eta^{2}} \\
0 & 1\end{array}\right)$ \\
\hline $\mathbf{M}_{\mathrm{OA}}^{\mathrm{Bs}}$ & $\left(\begin{array}{cc}1 & \frac{t \sqrt{1+\eta^{2}}}{2 \eta^{2}} \\
0 & 1\end{array}\right)$ & $\left(\begin{array}{cc}\frac{1}{\eta} & 0 \\
0 & \eta\end{array}\right)\left(\begin{array}{cc}1 & \frac{t \sqrt{1+\eta^{2}}}{2 \eta^{2}} \\
0 & 1\end{array}\right)$ \\
\hline $\mathbf{M}_{\mathrm{AB}}^{\mathrm{ni}}$ & $\left(\begin{array}{cc}1 & g-t / 2 \\
0 & 1\end{array}\right)$ & $\left(\begin{array}{cc}1 & g-t / 2 \\
0 & 1\end{array}\right)$ \\
\hline $\mathbf{M}_{\mathrm{AB}}^{\mathrm{B}}$ & $\left(\begin{array}{cc}1 & g-\frac{t}{\sqrt{1+\eta^{2}}} \\
0 & 1\end{array}\right)$ & $\left(\begin{array}{cc}1 & g-\frac{t}{\sqrt{1+\eta^{2}}} \\
0 & 1\end{array}\right)$ \\
\hline $\mathbf{M}_{\mathrm{BB}}$ & $\left(\begin{array}{cc}1 & 0 \\
-\frac{1}{f \cos \alpha} & 1\end{array}\right)$ & $\left(\begin{array}{cc}1 & 0 \\
-\frac{1}{f} \cos \alpha & 1\end{array}\right)$ \\
\hline $\mathbf{M}_{\mathrm{BE}}$ & $\left(\begin{array}{ll}1 & 2 v \\
0 & 1\end{array}\right)$ & $\left(\begin{array}{ll}1 & 2 v \\
0 & 1\end{array}\right)$ \\
\hline $\mathbf{M}_{\mathrm{EE}}$ & $\left(\begin{array}{cc}1 & 0 \\
-\frac{1}{f \cos \alpha} & 1\end{array}\right)$ & $\left(\begin{array}{cc}1 & 0 \\
-\frac{1}{f} \cos \alpha & 1\end{array}\right)$ \\
\hline $\mathbf{M}_{\mathrm{EF}}^{\mathrm{ni}}$ & $\left(\begin{array}{cc}1 & g-t / 2 \\
0 & 1\end{array}\right)$ & $\left(\begin{array}{cc}1 & g-t / 2 \\
0 & 1\end{array}\right)$ \\
\hline $\mathbf{M}_{\mathrm{EF}}^{\mathrm{B}}$ & $\left(\begin{array}{cc}1 & g-\frac{t}{\sqrt{1+\eta^{2}}} \\
0 & 1\end{array}\right)$ & $\left(\begin{array}{cc}1 & g-\frac{t}{\sqrt{1+\eta^{2}}} \\
0 & 1\end{array}\right)$ \\
\hline $\mathbf{M}_{\mathrm{FO}}^{\mathrm{ni}}$ & $\left(\begin{array}{cc}1 & \frac{t}{2 \eta} \\
0 & 1\end{array}\right)$ & $\left(\begin{array}{cc}1 & \frac{t}{2 \eta} \\
0 & 1\end{array}\right)$ \\
\hline $\mathbf{M}_{\mathrm{FO}}^{\mathrm{Bt}}$ & $\left(\begin{array}{cc}1 & \frac{t \sqrt{1+\eta^{2}}}{2 \eta^{2}} \\
0 & 1\end{array}\right)\left(\begin{array}{cc}\eta & 0 \\
0 & \frac{1}{\eta}\end{array}\right)$ & $\left(\begin{array}{cc}1 & \frac{t \sqrt{1+\eta^{2}}}{2 \eta^{2}} \\
0 & 1\end{array}\right)$ \\
\hline $\mathbf{M}_{\mathrm{FO}}^{\mathrm{Bs}}$ & $\left(\begin{array}{cc}1 & \frac{t \sqrt{1+\eta^{2}}}{2 \eta^{2}} \\
0 & 1\end{array}\right)$ & $\left(\begin{array}{cc}1 & \frac{t \sqrt{1+\eta^{2}}}{2 \eta^{2}} \\
0 & 1\end{array}\right)\left(\begin{array}{cc}\eta & 0 \\
0 & \frac{1}{\eta}\end{array}\right)$ \\
\hline
\end{tabular}

${ }^{a}$ Where they differ, matrices are given separately for normal incidence (ni) and Brewster's angle (B) crystal inclination in the tangential (Bt) or sagittal (Bs) direction.

Brewster's angled crystal cut is chosen will probably depend upon the availability of anti-reflection coatings for the normal incidence case.
To calculate the optimal beam for harmonic generation, we need to know the optical path length within the crystal. For a plate of thickness $t$ 
and refractive index $\eta_{1}$ inclined at an angle $\theta$ in a medium of index $\eta_{2}$, the ray length between the two faces is

$l=\frac{\eta_{1} t}{\sqrt{\eta_{1}^{2}-\eta_{2}^{2} \sin ^{2} \theta}}$.

For the specific case of Brewster's angle $\left(\tan \theta=\eta_{1} / \eta_{2}\right)$, we have

$l=\frac{t \sqrt{\eta_{1}^{2}+\eta_{2}^{2}}}{\eta_{1}}$

while at normal incidence, the corresponding length is simply $l=t$.

\subsection{Determining the required resonator beam}

Having chosen the crystal, we calculate the optimal fundamental beam $[6,8] .{ }^{1}$ Generally elliptical, this is characterized in the tangential and sagittal planes by the complex Gaussian beam parameters $q_{x, y}(z)$, defined at the foci by

$q_{x, y}=\frac{\mathrm{i} b_{x, y}}{2}$,

where

$b_{x, y}=\frac{2 \pi w_{x, y}^{2} \eta}{\lambda_{0}}$

are the confocal parameters, given in terms of the Gaussian beam waist radii $w_{x, y}$, the vacuum wavelength $\lambda_{0}$ and the refractive index $\eta$. The chosen crystal parameters define the optimal beam through the ratio $\xi_{x, y}=l / b_{x, y}$.

The beam undergoes refraction at the crystal faces, and from the outside hence appears to have differently sized and positioned foci. We now calculate the effective confocal parameters $b_{x, y}^{\prime \prime}$ and focal positions for both planes.

If the required beam waist at the crystal centre is described, for real $b$, by

\footnotetext{
${ }^{1}$ In the case of frequency quadrupling, the first doubling stage should be designed according to the recipe for parametric generation (see Ref. [9]), in order to maximize the power emitted into the elliptical Gaussian mode of the second stage.
}

$q=\frac{\mathrm{i} b}{2}$

and the appropriate matrix $\mathbf{M}_{\mathrm{OA}}$, which describes propagation from the crystal centre to its face and subsequent refraction, has elements $A, B, C$ and $D$, then the beam leaving the crystal face, characterized by

$q^{\prime} / \eta_{2}=\frac{A q / \eta_{1}+B}{C q / \eta_{1}+D}$

could equivalently result from the free propagation of a beam whose waist is described by

$q^{\prime \prime}=\frac{\mathrm{i} b^{\prime \prime}}{2}$

through a distance $p$ (and refractive index $\eta_{2}$ ) to the same point. With reference to Eq. (A.4), we may write

$q^{\prime}=q^{\prime \prime}+p$

and thus

$p=\operatorname{Re}\left(q^{\prime}\right)$

$b^{\prime \prime}=\frac{2}{\mathrm{i}} \operatorname{Im}\left(q^{\prime}\right)$.

Using the matrices given in Table 1 with the true beam parameters $b_{x}$ and $b_{y}$ at the crystal (primary) focus, we hence derive equivalent freespace parameters $b_{x}^{\prime \prime}$ and $b_{y}^{\prime \prime}$ and the corresponding equivalent focus-crystal face distances $p_{x}$ and $p_{y}$, which for later convenience we write in terms of the mean $p$ and astigmatic focal shift $z$ as $p_{x}=p+z / 2$ and $p_{y}=p-z / 2$. For the generic case in which the crystal is inclined at an arbitrary angle $\theta$, we obtain

$$
\begin{aligned}
& p=\frac{t}{4} \frac{\eta_{2}\left(2 \eta_{1}^{2}-\left(\eta_{1}^{2}+\eta_{2}^{2}\right) \sin ^{2} \theta\right)}{\left(\eta_{1}^{2}-\eta_{2}^{2} \sin ^{2} \theta\right)^{3 / 2}}, \\
& |z|=\frac{t}{2} \frac{\eta_{2}\left(\eta_{1}^{2}-\eta_{2}^{2}\right) \sin ^{2} \theta}{\left(\eta_{1}^{2}-\eta_{2}^{2} \sin ^{2} \theta\right)^{3 / 2}}, \\
& b^{\prime \prime}=\eta_{1} \eta_{2} \frac{1-\sin ^{2} \theta}{\eta_{1}^{2}-\eta_{2}^{2} \sin ^{2} \theta} b,
\end{aligned}
$$


where $z$ is positive if the crystal is inclined sagittally with respect to the mirrors and negative if inclined tangentially, and where $b^{\prime \prime}=\left(\eta_{2} / \eta_{1}\right) b$ in the direction in which the crystal is not inclined. The mean total distance $u$ between the concave mirror and the apparent crystal foci is given by $u=p+s$, where $s$ is the distance from the mirror to the crystal face; the tangential and sagittal crystal focus distances are thus $u_{x}=u+z / 2$ and $u_{y}=u-z / 2$ respectively. Expressions for $b_{x, y}^{\prime \prime}, p$ and $z$ for our three specific configurations are given in Table 2.

The cavity, which is now defined by the mirror focal length $f$, its incidence angle $\alpha$, the astigmatism $z$ and the mean distance between the mirror and the crystal focus $u$, is now examined separately in the tangential and sagittal directions as if the crystal were absent. Our aim is to find conditions under which the images of the chosen beam radii coincide to form a secondary focus at a distance $v$ from the mirror and are in the same proportion as the axes of the elliptical laser beam.

To simplify subsequent calculation, we convert the mirror object and image distances and the confocal parameters to dimensionless quantities by scaling according to the focal length. Thus $b_{x, y}^{\prime \prime}$ become $X, Y=b_{x, y}^{\prime \prime} / 2 f, z$ becomes $Z=z / 2 f$ and $u$ and $v$ are rewritten as $U=u / f$ and $V=v / f$. We also introduce the parameter $\phi=\cos \alpha$. Beam waists are already scaled in terms of the wavelength $\lambda_{0}$ by our use of the confocal parameter $b$. Finally, we define a secondary waist ellipticity parameter $\mathbf{e}=w_{y 2} / w_{x 2}$, where $w_{x 2, y 2}$ are the secondary waist radii. The true and reduced parameters used in our analysis are summarized in Table 3.

Table 2

Effective beam parameters and positions for normal incidence and for tangential and sagittal inclination at Brewster's angle

\begin{tabular}{lllll}
\hline & $b_{x}^{\prime \prime}$ & $p$ & $z$ & $b_{y}^{\prime \prime}$ \\
\hline ni & $b_{x} / \eta$ & $t / 2 \eta$ & 0 & $b_{y} / \eta$ \\
Bt & $b_{x} / \eta^{3}$ & $\frac{t}{4 \eta^{4}}\left(1+\eta^{2}\right)^{3 / 2}$ & $-\frac{t}{2 \eta^{4}} \sqrt{1+\eta^{2}}\left(\eta^{2}-1\right)$ & $b_{y} / \eta$ \\
& & & & \\
Bs & $b_{x} / \eta$ & $\frac{t}{4 \eta^{4}}\left(1+\eta^{2}\right)^{3 / 2}$ & $+\frac{t}{2 \eta^{4}} \sqrt{1+\eta^{2}}\left(\eta^{2}-1\right)$ & $b_{y} / \eta^{3}$ \\
\hline
\end{tabular}

Table 3

True and reduced parameters used for calculation

\begin{tabular}{|c|c|c|c|}
\hline & Property & True & Scaled \\
\hline \multirow[t]{2}{*}{ Scale by } & $\begin{array}{l}\text { Fundamental air } \\
\text { wavelength }\end{array}$ & $\lambda_{0}$ & \\
\hline & $\begin{array}{l}\text { Focal length at } \\
\text { normal incidence }\end{array}$ & $f$ & \\
\hline \multirow[t]{4}{*}{ Define } & $\begin{array}{l}\text { Tangential confocal } \\
\text { parameter }\end{array}$ & $b_{x}^{\prime \prime}$ & $X=b_{x}^{\prime \prime} / 2 f$ \\
\hline & $\begin{array}{l}\text { Sagittal confocal } \\
\text { parameter }\end{array}$ & $b_{y}^{\prime \prime}$ & $Y=b_{y}^{\prime \prime} / 2 f$ \\
\hline & Astigmatism & $z=p_{x}-p_{y}$ & $Z=z / 2 f$ \\
\hline & $\begin{array}{l}\text { Secondary waist } \\
\text { ellipticity parameter }\end{array}$ & $\mathbf{e}=w_{y 2} / w_{x 2}$ & \\
\hline \multirow[t]{3}{*}{ Solve for } & $\begin{array}{l}\text { Mirror incidence } \\
\text { angle }\end{array}$ & $\alpha$ & $\phi=\cos \alpha$ \\
\hline & $\begin{array}{l}\text { Mean distance to } \\
\text { crystal focus }\end{array}$ & $u$ & $U=u / f$ \\
\hline & $\begin{array}{l}\text { Distance to } \\
\text { secondary focus }\end{array}$ & $v$ & $V=v / f$ \\
\hline
\end{tabular}

\subsection{Solving the resonator equations}

To find the dimensions $(u, v, \alpha)$ of a cavity giving the required parameters $b_{x}^{\prime \prime}, b_{y}^{\prime \prime}, z$, secondary waist ellipticity parameter $\mathbf{e}$ and mirror focal length $f$, we refer to the function

$\mathscr{F}(u, v)=\left(\frac{u-1}{v-1}\right)(1-(u-1)(v-1))$,

shown in Fig. 4. This is simply the equation which, for normal incidence and in the absence of astigmatism, defines the radius $w$ of the waist which lies a distance $d_{1}$ from a concave mirror of focal length $f$ when the secondary waist distance is required to be $d_{2}$ :

$\left(\frac{\pi w^{2}}{\lambda_{0} f}\right)^{2}=\mathscr{F}\left(\frac{d_{1}}{f}, \frac{d_{2}}{f}\right)$.

The region of positive $\mathscr{F}(u, v)$, for which contours are shown, corresponds to the familiar range of cavity stability. It should be noted that the axis labelling is not identical to that on the well-known diagram for a two-mirror laser cavity; the case addressed here would correspond instead to a linear resonator of plane mirrors with a single intracavity lens.

In terms of the function $\mathscr{F}(u, v)$, then, our aim is the simultaneous solution of 


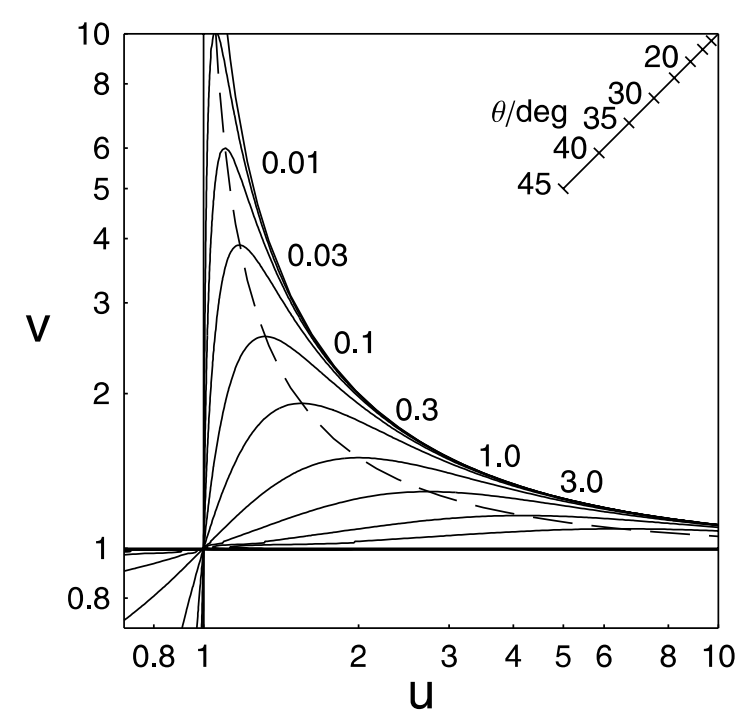

Fig. 4. Logarithmic plot of $\mathscr{F}(u, v)$ for graphical solution of astigmatic cavity design, showing the normalized principal waist size as a function of cavity geometry. The dashed line is the locus of the principal mode maxima.

$\mathscr{F}\left(\frac{U+Z}{\phi}, \frac{V}{\phi}\right)=\frac{X^{2}}{\phi^{2}}$,

$\mathscr{F}((U-Z) \phi, V \phi)=Y^{2} \phi^{2}$,

$\frac{\mathscr{F}(V / \phi,(U+Z) / \phi)}{\mathscr{F}(V \phi,(U-Z) \phi)}=\frac{1}{\mathbf{e}^{4} \phi^{4}}$

which respectively require specific values for the crystal waist radii in the tangential and sagittal directions and the secondary waist ellipticity. Given values for $X, Y, Z$ and $\mathbf{e}$, these equations allow us to find values of $U, V$ and $\phi$.

A first approximation can sometimes be found graphically. For the example of normal incidence $(Z=0)$, the solution to the first two equations lies at the intersection of the appropriate contours, displaced according to $\alpha$ along the $45^{\circ}$ line shown. Solution of the third equation involves evaluation of the function at the reflection in this $45^{\circ}$ line of the point of intersection, the process being repeated for a range of $\phi$ until the required value is found.

In the more general case, numerical solution is necessary. For given values of $X, Y, Z$ and $\phi$, Eqs. (16) and (17) may be combined to yield a quartic equation for $U$, which we give in Appendix B; varying $\phi$ then allows us to achieve the required ellipticity e. In most practical cases, there will be a large range of $f$ (and a corresponding range of $\alpha$ ) for which solutions are possible.

\subsection{Extracting the cavity dimensions}

Having found values for the scaled parameters $U, V$ and $\phi$, we may immediately write down the dimensions $u, v$ and $\alpha$ upon which they are based. For practical application, however, these must be converted into the real dimensions $g, c, d$ and $h$ shown in Figs. 1-3.

The distance $g$ from the crystal centre to the concave mirror is given by $g=s+j=u+j-p$, where $p$ has already been calculated in Eq. (11) and $j$ is given by

$$
\begin{aligned}
j & =\frac{l}{2} \cos \left(\theta-\theta^{\prime}\right) \\
& =\frac{t}{2} \frac{\eta_{2} \sin ^{2} \theta+\sqrt{\left(1-\sin ^{2} \theta\right)\left(\eta_{1}^{2}-\eta_{2}^{2} \sin ^{2} \theta\right)}}{\sqrt{\eta_{1}^{2}-\eta_{2}^{2} \sin ^{2} \theta}} .
\end{aligned}
$$

For the specific case of Brewster's angle $\left(\tan \theta=\eta_{1} / \eta_{2}\right)$, we have

$j=\frac{t \eta_{2}}{\sqrt{\eta_{1}^{2}+\eta_{2}^{2}}}$,

and thus

$g=u+t \frac{\left(3 \eta^{4}-2 \eta^{2}-1\right)}{4 \eta^{4} \sqrt{\eta^{2}+1}}$,

while at normal incidence we have simply $2 j=l=t$ and hence

$g=u+t \frac{\eta-1}{2 \eta}$.

The lateral displacement $c$ of the external rays from the crystal centre in the both Brewster's angled cases is given by

$c=\frac{t}{2} \frac{\sin \left(\theta-\theta^{\prime}\right)}{\cos \theta^{\prime}}=\frac{t}{2} \frac{\eta^{2}-1}{\eta \sqrt{\eta^{2}+1}}$.

For a bow-tie resonator, the transverse displacement of the plane mirrors (and thus the 
secondary beam waist) from the crystal centre is given simply by

$d=(v+g) \tan \alpha$

an alternative expression, which is also valid when the resonator forms a trapezium $(v \cos 2 \alpha<g)$, is

$d=\frac{1}{\sin 2 \alpha}(v-g \cos 2 \alpha-|v \cos 2 \alpha-g|)$.

If $v<g$, then the plane mirrors will be closest to the crystal, and the concave mirrors will thus enclose the secondary focus. In the configuration shown, the plane containing this secondary beam waist will be displaced from that containing the crystal centre by a distance

$h=\frac{c}{\tan \alpha}$.

\section{Discussion}

Although few authors give enough details to allow complete analysis of their resonators - there is a particular confusion as to whether beam parameters apply within or outside the crystal - a brief survey allows some general observations and a little practical advice.

\subsection{Cavity dimensions}

Most researchers have chosen configurations which correspond to the narrowing upper part of the region of cavity stability in Fig. 4. Typically, $U$ takes a value between 1.03 and 1.2 , while $V$ is in the range from 5 to 10; the mirror incidence angle $\alpha$ is between $5^{\circ}$ and $20^{\circ}$, and $X$ is much less than unity.

Various considerations lead to such choices. Firstly, the elliptical laser beam is usually made roughly circular before being matched into the cavity. A circular waist is then often chosen at the crystal, so that the optimal confocal parameter in both tangential and sagittal directions is smaller than the crystal length [8]. As shown in Table 2, the confocal parameter of the equivalent freespace beam will be smaller still, by at least a factor of $\eta$. To allow a reasonable space around the crystal, we usually find $t \ll 2 g$; this also accom- modates standard choices, mostly between 2 and 5 $\mathrm{cm}$, of the mirror focal length - a free parameter which, provided always that solutions are possible, may be chosen according to the designer's whim. The scaled confocal parameters $X, Y$ and astigmatism $Z$ are thus all much less than unity, $U$ is close to 1 and the mirror incidence angle $\alpha$ is relatively small.

Using the procedure outlined in this paper, the ellipticity of the secondary waist may be matched directly to that of the laser emission: whilst there may not always be a convenient solution, there is no fundamental constraint. Further, as we have shown elsewhere [6], the optimal beam for harmonic generation is often itself elliptical (and tolerant of a degree of ellipticity), in which case the larger of the corresponding confocal parameters may approach cavity dimensions.

There are some advantages in moving to smaller cavities which, as noted by Ritter et al. [10], offer better mechanical and thermal stability. The free spectral range of the resonator, which is often used to control the laser wavelength, is increased, as is the absolute line width of the cavity resonance. Further, if the resonator is to be varied in temperature, evacuated or flooded with dry nitrogen, then the smaller size is generally helpful. One important class of design which indeed falls well into the smaller regime is that of the monolithic resonator [11], in which the cavity mirrors are formed by the faces of the crystal itself. For such cavities, the mirror separation, curvature radius and beam confocal parameters are all similar; $U$ and $V$ are typically around 1.3 .

\subsection{Mirror curvature and incidence angle}

The freedom so far to make an arbitrary choice for either the mirror focal length or incidence angle allows us to take into account higher-order effects such as coma. This has been treated using geometrical optics by Dunn and Ferguson [12], who found that for $b \ll l$, coma due to the off-axis mirror and a crystal face inclined tangentially at Brewster's angle cancel when

$f \sin \alpha=t \frac{\left(\eta^{2}+1\right)^{1 / 2}\left(\eta^{4}-1\right)}{\eta^{7}}$ 
provided that the crystal face is inclined in the direction that takes it closer to being parallel to the mirror.

This latter requirement is not satisfied by the common arrangement in which a crystal with parallel faces is placed within the bow-tie resonator of Fig. 1, but may indeed be met by the alternative cavities shown in Fig. 3. In cavity (a), the crystal faces are inclined in opposite directions $[13,14] ; g$ and $c$ are as given in Eqs. (21) and (23), while $h=0$ and

$d=\left(v+g^{\prime}\right) \tan \left(\alpha-\left(\theta-\theta^{\prime}\right) / 2\right)$,

where $g^{\prime}=g \cos \left(\theta-\theta^{\prime}\right)+c \sin \left(\theta-\theta^{\prime}\right)$. Alternatively, as in cavity (b), it may be the mirrors that are oppositely inclined [15-17]; $g$ and $c$ are again unchanged, and the plane mirror incidence angle is given by

$\beta=\tan ^{-1} \frac{c \cos 2 \alpha-g \sin 2 \alpha}{c \sin 2 \alpha+g \cos 2 \alpha-v}$.

If coma may be neglected and the secondary waist ellipticity is unimportant, then both $f$ and $\phi=\cos \alpha$ will be free parameters, and we may instead choose the configuration in which the mode size is least sensitive to mirror position, which for small $\mathscr{F}$ may be considered to occur when the tangential and sagittal beam waists are simultaneous maxima as functions of the concave mirror separation [18]. The calculation of this configuration is described in Appendix C.

\subsection{Mirrors and coupling}

Apart from their radii of curvature or focal lengths, the mirrors are defined by their reflectivities which, together with crystal and other optical losses, determine the enhancement factor of the resonator: their role has been thoroughly analysed elsewhere $[19,20]$, and we shall not discuss it further here. Usually, three of the mirrors are highly reflecting, the fourth having a transmission of 1$2 \%$ by which to couple in the fundamental radiation. Because the cavity losses, and thus optimal mirror reflectivity, cannot be known until the cavity is tested, several researchers have suggested using highly reflecting mirrors throughout and controlling the enhancement factor via a separate variable optical coupler [20,21].

At least one of the mirrors should be highly transmitting at the wavelength of the harmonic to allow it to be extracted. To avoid cylindrical focussing, it is better to couple light into and out of the resonator through the plane mirrors. Astigmatism in the fundamental and harmonic beams can be removed by slightly tilting the input focussing and output collimation lenses.

The optical mounts used should be stable, free of backlash and offer fine adjustment, and the flexure type [22,23] is often chosen ${ }^{2}$. As always, it is helpful to decouple rotational and translational adjustments. The mounts can usually be positioned according to their calculated locations and aligned using the fine adjustments alone [24]. We find a small CCD video camera to be invaluable during alignment: with the commercial lens removed, both fundamental and harmonic wavelengths can usually be detected.

\section{Acknowledgements}

T.F. is grateful for support from the UK Engineering and Physical Sciences Research Council and the European Union in the course of this work, and to Dr. Grant Ritchie for checking some of the equations.

\section{Appendix A. Ray-transfer matrices}

In the following section, we give the ray-transfer matrices that describe the resonator. Although we do not use all of them for the design of the cavity, they are useful for checking the results and for determining, for example, the beam intensities at the mirrors and crystal faces. We therefore give them in full.

\footnotetext{
${ }^{2}$ Although nickel silver has been suggested for flexure mounts (see Ref. [24]) because of its high tensile strength to Young's modulus ratio, many high-tensile alloys of aluminium possess similar properties, particularly when tempered. More readily available and easy to machine, they can be anodised to give a harder surface and a convenient black finish.
} 
The matrices describe the propagation of an optical ray, characterized by its displacement $r$ from, and angle $\mathrm{d} r / \mathrm{d} z$ to, the optical axis. We write these as a column vector,

$\mathbf{R}=\left(\begin{array}{c}r \\ \eta \frac{\mathrm{d} r}{\mathrm{~d} z}\end{array}\right)$

In this form [7], the Gaussian beam waist is transformed according to

$\frac{q_{2}}{\eta_{2}}=\frac{A q_{1} / \eta_{1}+B}{C q_{1} / \eta_{1}+D}$

where $A, B, C$ and $D$ are as usual the elements of the appropriate ray-transfer matrix and the Gaussian beam parameter $q$ is defined by

$\frac{1}{q}=\frac{1}{R}-\mathrm{i} \frac{\lambda_{0}}{\pi \omega^{2} \eta}=\frac{1}{R}-\frac{2 \mathrm{i}}{b}$,

$\lambda_{0}$ being the fundamental wavelength in vacuo, $R$ and $w$ respectively the radius of curvature and Gaussian beam waist radius, $\eta$ the relevant refractive index and $b$ the beam confocal parameter.

\section{A.1. Free propagation}

The ray-transfer matrix for propagation through a distance $z$ in a medium with a refractive index $\eta$ is

$\mathbf{M}=\left(\begin{array}{cc}1 & z / \eta \\ 0 & 1\end{array}\right)$

\section{A.2. Refraction at an angled plate}

Refraction at an interface inclined at $\theta_{1}$ to the incident ray and $\theta_{2}$ to the refracted ray is described by the matrix

$$
\mathbf{M}=\left(\begin{array}{cc}
\frac{\cos \theta_{2}}{\cos \theta_{1}} & 0 \\
0 & \frac{\cos \theta_{1}}{\cos \theta_{2}}
\end{array}\right) .
$$

For a plate of thickness $t$ and refractive index $\eta_{1}$ inclined at Brewster's angle within a medium of index $\eta_{2}$, the matrix describing transmission in the tangential plane of the crystal is thus, with reference to Eq. (2),

$$
\begin{aligned}
& \mathbf{M}=\left(\begin{array}{cc}
\frac{\eta_{2}}{\eta_{1}} & 0 \\
0 & \frac{\eta_{1}}{\eta_{2}}
\end{array}\right)\left(\begin{array}{cc}
1 & \frac{t \sqrt{\eta_{1}^{2}+\eta_{2}^{2}}}{\eta_{1}^{2}} \\
0 & 1
\end{array}\right)\left(\begin{array}{cc}
\frac{\eta_{1}}{\eta_{2}} & 0 \\
0 & \frac{\eta_{2}}{\eta_{1}}
\end{array}\right), \\
& \mathbf{M}=\left(\begin{array}{cc}
1 & \frac{t \eta_{2}^{2} \sqrt{\eta_{1}^{2}+\eta_{2}^{2}}}{n_{1}^{4}} \\
0 & 1
\end{array}\right) .
\end{aligned}
$$

This may be broken into two matrices accounting respectively for propagation from the entrance face to the plate centre and from the centre to the exit face:

$$
\begin{aligned}
& \mathbf{M}_{\mathrm{FO}}=\left(\begin{array}{cc}
1 & \frac{t \sqrt{\eta_{1}^{2}+\eta_{2}^{2}}}{2 \eta_{1}^{2}} \\
0 & 1
\end{array}\right)\left(\begin{array}{cc}
\frac{\eta_{1}}{\eta_{2}} & 0 \\
0 & \frac{\eta_{2}}{\eta_{1}}
\end{array}\right), \\
& \mathbf{M}_{\mathrm{OA}}=\left(\begin{array}{cc}
\frac{\eta_{2}}{\eta_{1}} & 0 \\
0 & \frac{\eta_{1}}{\eta_{2}}
\end{array}\right)\left(\begin{array}{cc}
1 & \frac{t \sqrt{\eta_{1}^{2}+\eta_{2}^{2}}}{2 \eta_{1}^{2}} \\
0 & 1
\end{array}\right) .
\end{aligned}
$$

Propagation in the sagittal plane of the crystal is described simply by the matrix for free propagation through the distance $l$ in a medium of index $\eta_{1}$,

$\mathbf{M}=\left(\begin{array}{cc}1 & \frac{t \sqrt{\eta_{1}^{2}+\eta_{2}^{2}}}{n_{1}^{2}} \\ 0 & 1\end{array}\right)$.

Astigmatism is thus introduced because the crystal differs in apparent thickness between the tangential and sagittal planes. The maximum difference of $0.449 t$ occurs at $\eta_{1} / \eta_{2}=1.60$.

\section{A.3. Off-axis spherical mirror}

The ray-transfer matrices for a spherical mirror of focal length $f$ (and hence curvature radius $R=2 f$ ) at an angle $\alpha$ to the beam axis are, in the mirror's tangential plane,

$$
\mathbf{M}_{\mathrm{T}}=\left(\begin{array}{cc}
1 & 0 \\
-\frac{\eta}{f \cos \alpha} & 1
\end{array}\right)
$$


and in its sagittal plane,

$\mathbf{M}_{\mathrm{S}}=\left(\begin{array}{cc}1 & 0 \\ -\frac{\eta}{f} \cos \alpha & 1\end{array}\right)$.

Using the above results, we may now write down the transfer matrices for successive stages of the cavity round-trip, as summarized in Table 1. For clarity, components have not always been combined into a single product matrix; where they depend upon the crystal orientation, the respective matrices are given separately.

\section{Appendix B. Solution for $U$}

For given values of $X, Y, Z$ and $\phi$-as, for example, when we have defined the target crystal beam waists and astigmatism and have made an arbitrary choice of mirror focal length-Eqs. (16) and (17) may be combined to yield a quartic equation in $U$ as a function of the mirror incidence angle via $\phi=\cos \alpha$. Since this is a rather messy process, we give the result obtained using Mathematica [25] below.

$$
\begin{aligned}
0= & U^{4}\left(\phi^{3}-\phi\right)+U^{3}\left(1-\phi^{4}\right) \\
+ & U^{2}\left(\phi^{4}(X+Z)+\phi^{3}\left(1-2 Z^{2}\right)\right. \\
& \left.+\phi^{2}(-X+Y-4 Z)+\phi\left(-1+2 Z^{2}\right)-Y+Z\right) \\
+ & U\left(\phi^{4} Z(-2 X+Z)-\phi^{3}(2 X+Y-2 Z)\right. \\
& +2 \phi^{2}(X+Y) Z+\phi(X+2(Y+Z)) \\
& -Z(2 Y+Z)) \\
+ & \left(\phi^{4}(X-Z) Z^{2}\right. \\
& +\phi^{3}\left(X(Y+2 Z)+Z\left(-Y-3 Z+Z^{3}\right)\right) \\
+ & \phi^{2}\left(X-Y-2 Z-X Z^{2}+Y Z^{2}+4 Z^{3}\right) \\
- & \phi\left(X(Y+Z)+Z\left(-2 Y-3 Z+Z^{3}\right)\right) \\
& \left.-Z^{2}(Y+Z)\right) .
\end{aligned}
$$

The solution to this equation may be inserted into Eq. (18) to establish the secondary waist ellipticity corresponding to this incidence angle and, by repeating the process for a range of $\phi$, an overall solution may thus be found.

\section{Appendix C. Solution for greatest stability}

It is apparent from Fig. 4 that the beam waist may be adjusted, by varying $u$, up to a maximum value determined by $v$ [26]. At these maxima, the mode size is least sensitive to mirror position and, for some researchers, simultaneous maxima of both tangential and sagittal mode sizes therefore provide the preferred configuration [18].

Contours of constant $\mathscr{F}(u, v)$, corresponding to a given waist size, have a common form in a linear plot, being scaled about the point $(1,1)$ by a factor of $\sqrt{\mathscr{F}}$ in $u$ and its reciprocal in the $v$ direction. The turning points of these curves $\left((\partial \mathscr{F} / \partial u)_{v}=0\right)$ describe a locus, shown dashed in Fig. 4,

$(u-1)(v-1)=\frac{1}{2}$

with $\mathscr{F}=(u-1)^{2}$. For the $u$ and $v$ coordinates to coincide for both the tangential and sagittal directions, we find

$2 Z+Y-X=\phi-\frac{1}{\phi}$,

$\frac{1}{2 X} \phi^{4}+\phi^{3}-\phi-\frac{1}{2 Y}=0$.

Substituting for $X, Y$ and $Z$ from Table 3, we seek a focal length $f$ that will simultaneously satisfy these equations. If both cavities lie in the upper right quadrant of Fig. $4(u, v>1)$, this will be given by

$$
\begin{aligned}
f^{2} & =\frac{\left(2 z+b_{y}^{\prime \prime}-b_{x}^{\prime \prime}\right) b_{x}^{\prime \prime} b_{y}^{\prime \prime} z}{\left(b_{x}^{\prime \prime}-b_{y}^{\prime \prime}\right)^{2}} \\
& \pm \frac{\sqrt{b_{x}^{\prime \prime} b_{y}^{\prime \prime}\left(b_{x}^{\prime \prime}+b_{y}^{\prime \prime}\right)^{2}\left(2 z+b_{y}^{\prime \prime}-b_{x}^{\prime \prime}\right)^{3}\left(2 z+b_{x}^{\prime \prime}-b_{y}^{\prime \prime}\right)}}{4\left(b_{x}^{\prime \prime}-b_{y}^{\prime \prime}\right)^{2}} .
\end{aligned}
$$

The sign of the $b_{x, y}^{\prime \prime}$ should be changed if the corresponding cavity instead lies in the opposite quadrant $(u, v<1)$. The mirror incidence angle may then be found by substituting this value back into Eq. (C.2) to obtain $\phi$. 


\section{References}

[1] H.W. Kogelnik, E.P. Ippen, A. Dienes, C.V. Shank, IEEE J. Quant. Electron. 8 (1972) 373-379.

[2] C.E. Dunn, M.H. Dunn, Optica Acta 28 (1981) 1413-1423.

[3] D.M. Kane, Opt. Commun. 71 (1989) 113-118.

[4] J.-P. Weber, IEEE J. Quant. Electron. 30 (1994) 24072416.

[5] X.G. Sun, G.W. Switzer, J.L. Carlsten, Appl. Phys. Lett. 76 (2000) 955-957 ${ }^{3}$.

[6] T. Freegarde, J. Coutts, J. Walz, D. Leibfried, T.W. Hänsch, J. Opt. Soc. Am. B 14 (1997) 2010-2016.

[7] A.E. Siegman, Lasers, University Science Books, California (1986). Non-planar resonators are discussed on p. 537; for Gaussian beam propagation, see p. 783; prototype matrices are given in Table 15.1, pp. 585-586.

[8] G.D. Boyd, D.A. Kleinman, J. Appl. Phys. 39 (1968) 35973639.

[9] D.J. Kuizenga, Appl. Phys. Lett. 21 (1972) 570-572.

[10] G. Ritter, S.-M. Bae, U. Eichmann, Appl. Phys. B 66 (1998) 609-612.

[11] W.J. Kozlovsky, W.P. Risk, W. Lenth, B.G. Kim, G.L. Bona, H. Jaeckel, D.J. Webb, Appl. Phys. Lett. 65 (1994) 525-527.

[12] M.H. Dunn, A.I. Ferguson, Opt. Commun. 20 (1977) 214 219.
[13] J.S. Nielsen, Opt. Lett. 20 (1995) 840-842.

[14] J.D. Bhawalkar, Y. Mao, H. Po, A.K. Goyal, P. Gavrilovic, Y. Conturie, S. Singh, Opt. Lett. 24 (1999) 823-825.

[15] J.C. Bergquist, H. Hemmati, W.M. Itano, Opt. Commun. 43 (1982) 437-442.

[16] A. Steinbach, M. Rauner, F.C. Cruz, J.C. Bergquist, Opt. Commun. 123 (1996) 207-214.

[17] T. Kaing, M. Houssin, Opt. Commun. 157 (1998) 155-160.

[18] F. Schmidt-Kaler, D. Leibfried, S. Seel, C. Zimmermann, W. König, M. Weitz, T.W. Hänsch, Phys. Rev. A 51 (1995) 2789-2800.

[19] Y. Kaneda, S. Kubota, Appl. Opt. 36 (1997) 7766-7775.

[20] K.R. Lykke, Opt. Commun. 157 (1998) 88-92.

[21] J. Torgerson, W. Nagourney, Opt. Commun. 161 (1999) 264-266.

[22] K.B. MacAdam, A. Steinbach, C. Wieman, Am. J. Phys. 60 (1992) 1098-1111.

[23] A. Nguyen, D.T. Cassidy, Rev. Sci. Instrum. 66 (1995) 4458-4460.

[24] M. de Angelis, G.M. Tino, P. De Natale, C. Fort, G. Modugno, M. Prevedelli, C. Zimmermann, Appl. Phys. B 62 (1996) 333-338.

[25] Mathematica software from Wolfram Research, Inc., Champaign, IL 61820-7237, USA.

[26] A. Hemmerich, D.H. McIntyre, C. Zimmermann, T.W. Hänsch, Opt. Lett. 15 (1990) 372-374.

\footnotetext{
${ }^{3}$ We note that Eq. (2) contain several errors, and should read $l_{x}=l+\frac{t \eta^{2}\left(1-\sin ^{2} \phi\right)}{\left(\eta^{2}-\sin ^{2} \phi\right)^{3 / 2}}-t \sqrt{1-\sin ^{2} \phi}-\frac{t \sin ^{2} \phi}{\sqrt{\eta^{2}-\sin ^{2} \phi}}$ and $l_{y}=l+\frac{t}{\sqrt{\eta^{2}-\sin ^{2} \phi}}-t \sqrt{1-\sin ^{2} \phi}-\frac{t \sin ^{2} \phi}{\sqrt{\eta^{2}-\sin ^{2} \phi}}$. $l_{x}$ and $l_{y}$ here correspond to $2 u_{x}$ and $2 u_{y}$ in this paper, with $\phi$ equivalent to our $\theta$.
} 